\title{
EWSR1-SMAD3 fibroblastic tumour of bone: expanding the clinical spectrum
} CASE REPORT

\author{
Solange De Noon ${ }^{1} \cdot$ Adrienne M Flanagan $^{1,2} \cdot$ Roberto Tirabosco $^{1} \cdot$ Paul O'Donnell $^{3,4} \cdot$ Fernanda Amary ${ }^{1,2}$
}

\begin{abstract}
EWSR1-SMAD3 fibroblastic tumour is a recently described soft tissue lesion. To date, eight cases have been reported, all sited in superficial soft tissue, typically occurring in the hands and feet with a tendency for local recurrence if incompletely excised. No metastatic spread has been reported, and hence, these tumours are currently considered benign. Herein, we present the radiological and histological features of the first reported occurrence of this entity in bone: a 44-year-old man with a tumour in the right tibia, treated with en bloc resection and showing no signs of relapse at 7 years. This tumour should be added to the differential diagnosis of bone lesions which harbour EWSRI gene rearrangement.
\end{abstract}

Keywords EWSR1 - EWSR1-SMAD3 - Bone tumour · Tumour · Sarcoma

\section{Introduction}

Novel bone and soft tissue tumours associated with specific genetic drivers are increasingly being identified, often with the aid of powerful modern techniques such as massive parallel sequencing [1]. Chromosomal translocation leading to a chimeric fusion defines many sarcomas. In addition to recurrent genomic alterations, these tumours also frequently demonstrate similar clinical and histological features with important implications for management and prognosis [2]. EWSR1 is recognised as the most frequently rearranged gene, involved in over ten subtypes of sarcoma in bone and soft tissue, in addition to a range of non-sarcoma malignancies [3]. In 2018, Kao et al. reported three soft tissue tumours sharing histological similarities and harbouring a novel EWSR1SMAD3 fusion [4]. Subsequent reports recapitulated these findings and provided further evidence of a distinct tumour

$\triangle$ Fernanda Amary

Fernanda.amary@nhs.net

Department of Histopathology, Royal National Orthopaedic Hospital, Stanmore, Greater London, UK

2 Cancer Institute, University College London, London, UK

3 Department of Radiology, Royal National Orthopaedic Hospital, Stanmore, UK

4 Institute of Orthopaedics and Musculoskeletal Science, University College London, London, UK type, which has been recently included in the 2020 World Health Organization classification of bone and soft tissue tumours [5]. To date, however, only 8 cases have been published in the English literature and much remains unknown about the histological, clinical and genomic features of this emerging tumour type. We present the first case of a tumour with this characteristic fusion arising in bone, with the aim of expanding the clinical, imaging and histopathological spectrum of fibroblastic bone lesions and underscoring the utility of next-generation sequencing in the diagnosis of sarcoma.

\section{Case report}

A 44-year-old male presented with a 9-month history of right knee pain and worsening night pain. On examination, diffuse swelling of the proximal right calf was noted, with retained movements at the joint and no obvious effusion. No neurological deficit was observed. A radiograph revealed a lytic lesion in the proximal tibia with both aggressive and non-aggressive features (Fig. 1), and a high-grade chondrosarcoma was considered. Magnetic resonance imaging (MRI) demonstrated an intramedullary lesion measuring approximately $8 \mathrm{~cm}$ in maximum dimension, with a large, lobular, predominantly posterior extraosseous component. The tumour showed a biphasic appearance on MRI (distinct central low T2 signal intensity with peripheral hyperintensity) and marked oedema-like high signal in the adjacent marrow and soft tissue (Fig. 2). A 


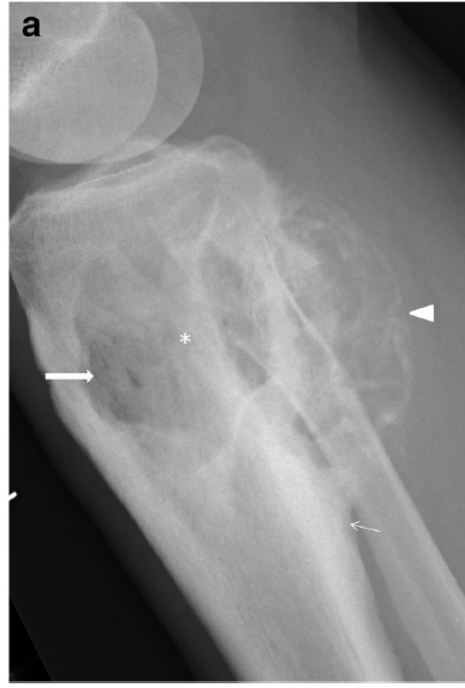

Fig. 1 Lateral (a) and anteroposterior (b) radiographs of the right proximal tibia in a 44-year-old man. A lobular medullary tumour showing geographic bone destruction (white*) is seen. The margin is well-defined and shows incomplete thin sclerosis, but there are small lucencies suggesting cortical permeation (block arrow in (a)). Cortex

chondrosarcoma, either dedifferentiated or mesenchymal (the latter in view of central hypointensity), was thought possible, but the appearances were not felt to be pathognomonic. Computed tomography confirmed an ossified margin around the extraosseous mass but no tumour matrix mineralisation. No neurovascular encasement or joint involvement was seen and there were no skip metastases.

A needle core biopsy mirrored the biphasic radiological appearance: there were predominantly paucicellular areas of hyalinised stroma with foci of cartilaginous differentiation, and cellular areas featuring uniform oval to spindled cells with scattered mitotic activity and areas of necrosis (Fig. 3 a-b). The diagnosis of an unusual cartilaginous tumour, raising the possibility of a chondrosarcoma (possibly dedifferentiated or mesenchymal) was first considered. No distant disease was identified on computed tomography of the chest or technetium isotope bone scan.

Proximal tibial replacement was the preferred surgical management, but as there was displacement and compression of the popliteal artery by the mass, amputation was a distinct possibility. A decision was made to treat with neoadjuvant chemotherapy in order to improve the chances of non-ablative resection. However, worsening symptoms after one cycle of doxorubicin and cisplatin prompted urgent surgical intervention, and the patient underwent a proximal tibial replacement, following which chemotherapy was not restarted. Histologically, the tumour was composed of nodular areas of hyalinised stroma admixed with cellular sheets of spindled to oval cells with moderate cytological atypia. A

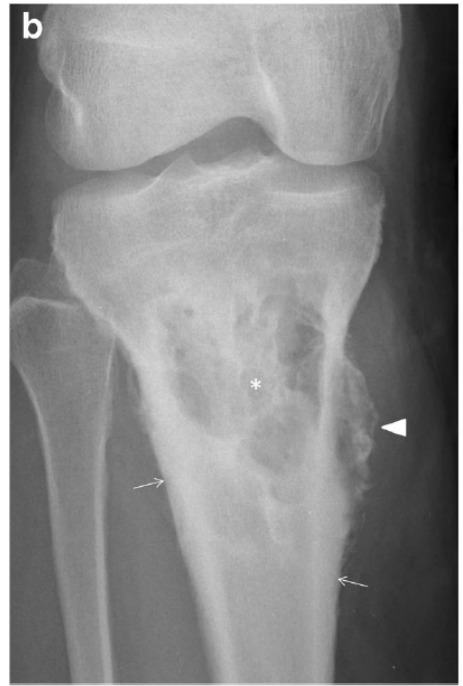

adjacent to the tumour is mildly thickened (thin arrows), with subtle irregular periosteal new bone formation, and there is mild expansile remodelling. An ossified, predominantly posterior extraosseous mass (arrowheads) is also seen

haemangiopericytic growth pattern was seen throughout. Within the hyalinised nodular areas, foci of myxoid change and myxochondroid differentiation were seen as well as a striking whorled pattern of hyalinisation (Fig. 3) Very few scattered cells expressed ETS-related gene (ERG). Immunohistochemistry for desmin, smooth muscle actin, epithelial membrane antigen, CD31, S100, and pancytokeratin MNF116 was negative. Given the unusual features, a descriptive diagnosis was given, again raising the possibility of an unusual form of chondrosarcoma. The tumour was clear of bone resection margins with the extraosseous component confined by attenuated periosteum and a shell of reactive bone. The bone margins were free of disease.

Given the uncertainty of the diagnosis, this case was submitted for whole-genome sequencing as part of the 100,000 Genomes Project [6]. This revealed the presence of a $\mathrm{t}(15 ; 22)(\mathrm{q} 22.33 ; \mathrm{q} 12.2)$ EWSR1-SMAD3 rearrangement. Subsequent fluorescence in situ hybridisation, using breakapart probes as previously described (EWSR1 break-apart probe, Vysis, Abbott, Maidenhead, UK) [7], confirmed an EWSR1 rearrangement. Whole-genome sequencing and standard bioinformatics analysis [6] revealed an otherwise relatively quiet genome with 5818 somatic nucleotide variants (SNVs) and small insertion/deletions (indels) detected and a low mutational burden (1.41 per megabase). No pathogenic SNVs or indels were identified in any recognised or actionable cancer-related genes. A non-validated ITGA10/MUC4 rearrangement was also identified.

The patient is well with no sign of relapse at 84 months. 
Fig. 2 MRI (a-c) and CT (d) of the right proximal tibia. Coronal T1-weighted MRI (a) shows a well-defined, heterogeneous but predominantly isointense tumour with a thin, incomplete low signal margin. There is subtle peripheral hyperintensity (white arrows) and ill-defined low signal in the adjacent marrow consistent with oedema. Sagittal T2-weighted (b) and axial proton density fat saturated (c) images show rather whorled, heterogeneous low signal within both the medullary and extraosseous components of the mass (white*), distinct from relatively hyperintense, predominantly peripheral tumour (thin vertical arrows). The posterior cortex is breached and remodelled adjacent to the extraosseous mass. Axial MRI and corresponding CT at the time of biopsy (d) show that the extraosseous component is traversed by an irregular bony septum (black*) and surrounded by an ossified rim (arrowhead)

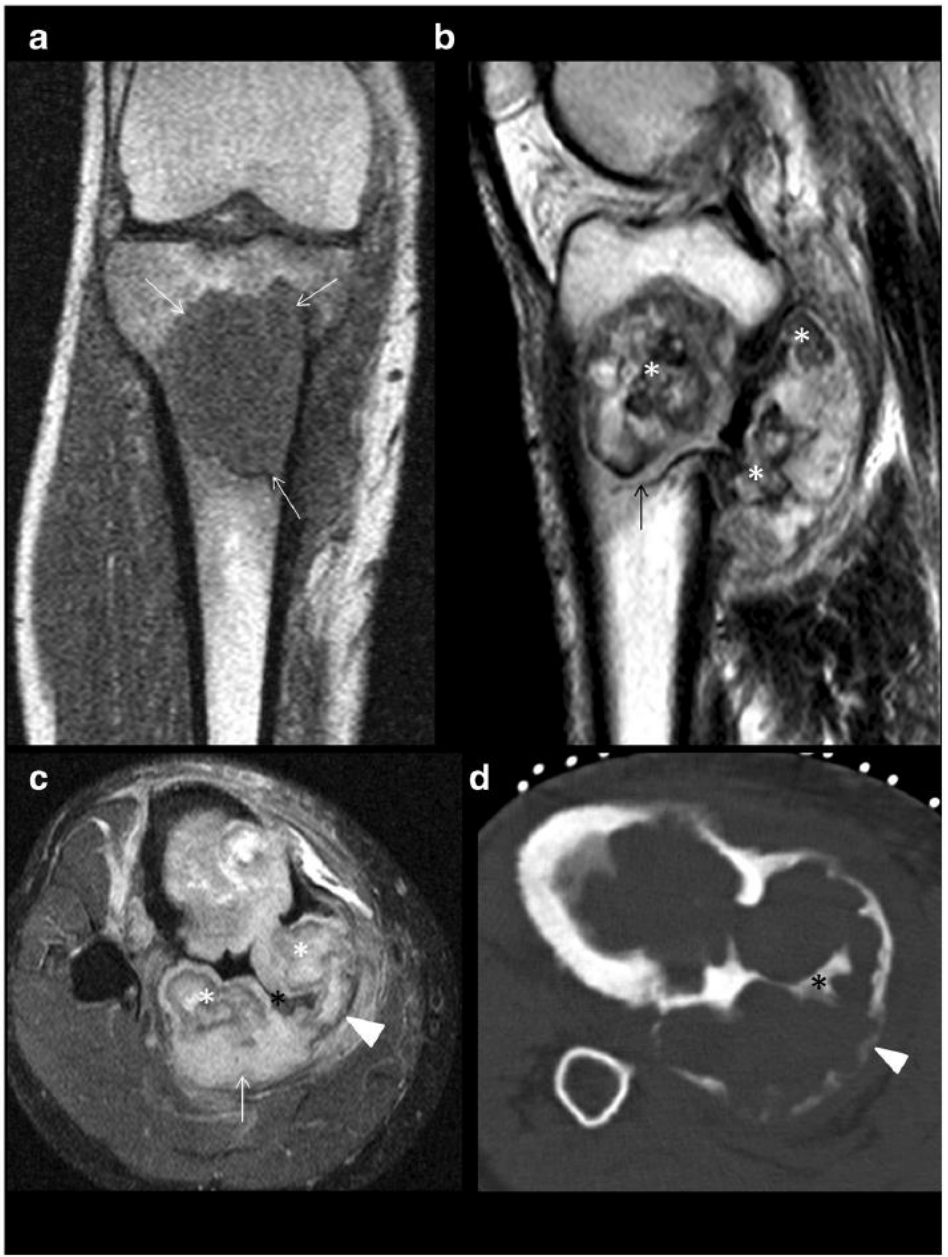

\section{Discussion}

EWSR1-SMAD3 positive fibroblastic tumour was first described as a distinct soft tissue lesion in 2018 [4]. Since this series of three tumours, five additional cases have been published, four with molecular confirmation [8, 9]. All are characterised by the presence of a biphasic, variably cellular zonal appearance with cellular, often peripheral areas of spindled to oval cells and central paucicellular to acellular hyalinised zones. The tumours have low mitotic activity and lack significant atypia. Cases occurred over a wide age, and all the tumours arose in dermal or superficial subcutaneous soft tissues in the extremities: 7 acral ( 5 in the foot and 2 in the hand) and one in the calf. Six patients were female and two were male. Four patients developed local recurrences, usually associated with incomplete excision.

We present the first case of EWSR1-SMAD3 fibroblastic tumour arising within bone, our case demonstrating several of the features previously described in soft tissue lesions, including a biphasic nodular growth pattern consisting of spindled to ovoid cells with admixed zones of hypocellular hyalinised and focally myxoid stroma. This tumour was relatively large $(8 \mathrm{~cm})$, showed aggressive radiological features and demonstrated focal cytological atypia and necrosis, and only very focal ERG nuclear expression. This contrasts with the bland cytological features and strong nuclear ERG immunopositivity reported in the previous cases. In addition, there were areas of chondroid differentiation not seen in the soft tissue counterpart, which resulted in the initial evaluation of this lesion as a cartilage-forming tumour and is a possible risk for the misdiagnosis of lesions in bone.

This destructive bone lesion showed both indolent and aggressive imaging features: slow growth was suggested by a well-demarcated, partially sclerotic tumour margin, sclerosis and thickening of the adjacent cortex and ossified septa traversing the tumour. More rapid growth was 
Fig. 3 Photomicrographs of the biopsy sample (a and $\mathbf{b}$ )

predominantly composed of paucicellular chondro-hyaline tissue and cellular areas of ovoid to spindled cells. Resection specimen $(\mathbf{c}-\mathbf{h})$ featuring the biphasic pattern with cellular areas composed of spindle to oval cells (e and $\mathbf{f}$ ) and myxo-hyaline to chondroid foci (c, $\mathbf{d}$ and $\mathbf{g}$ ) with a striking whorled pattern (h)


suggested by bone permeation, cortical destruction and an extraosseous mass. Computed tomography showed that the mass was enclosed by a shell of reactive bone-another indolent feature - and confirmed the absence of cartilaginous matrix mineralisation. A biphasic appearance was noted on MRI, with lobular, rather whorled low T2 signal intensity centrally, surrounded by a rim of relatively hyperintense tumour. Mesenchymal chondrosarcoma may also show biphasic appearances, with central low signal intensity due to calcification [10]. However, in our case there was no radiographic evidence of calcification, and the hypointense areas corresponded histologically to hypocellular hyalinised tumour (Fig. 4). The whorled central low T2 signal/peripheral hyperintensity seen in the current case also differs from the typical biphasic morphology of a dedifferentiated chondrosarcoma [11]. 
Fig. 4 Pathology-radiology correlation. Gross specimen and sagittal T2-weighted MR image.

Firm white lobular tumour within the proximal tibia and

extraosseous mass corresponds to the paucicellular hyalinised

component and is markedly hypointense on T2-weighted MRI sequences $(*)$
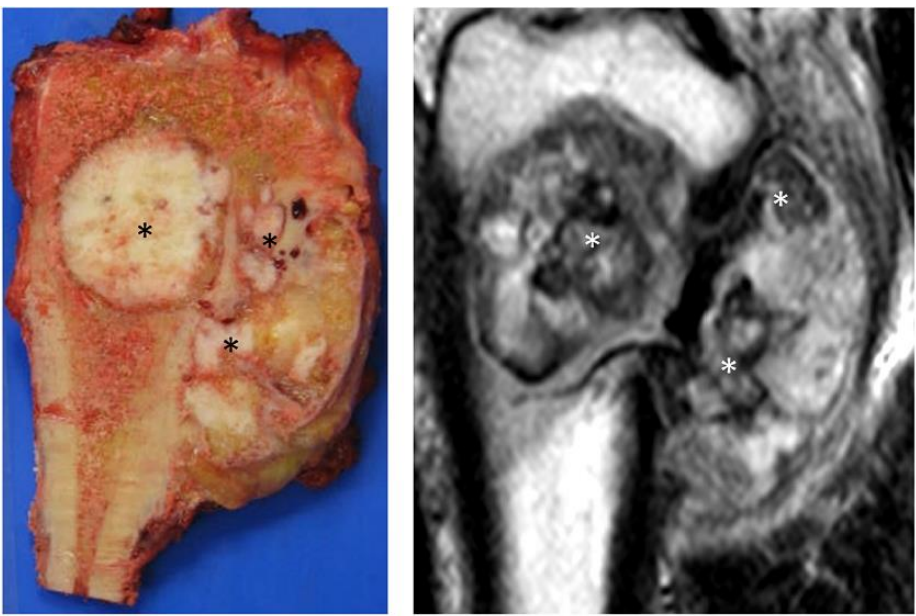

The soft tissue variant of this lesion is considered benign in the current World Health Organization classification of bone and soft tissue tumours [5], but the biological behaviour of the bone entity is as yet unknown. In our patient, the disease has remained localised with no relapse for 7 years. However, based on a combination of the pathology and imaging features at presentation, we cannot exclude the possibility of 'locally aggressive' behaviour. This tumour was treated with en bloc excision, but it is uncertain if curettage would have been appropriate. In addition, other tumours (for example epithelioid haemangioma) tend to be locally aggressive in bone while considered benign in soft tissue.

EWSR1 has been well-documented as a common gene partner in several driver fusions across a range of tumour types, including benign and malignant mesenchymal tumours as well as some carcinomas [12]. EWSR1 typically contributes its transactivation domain to the chimeric protein, resulting in deregulation expression of the $3^{\prime}$ gene partner and its downstream targets. The SMAD3 gene is a transcriptional regulator that forms part of the transforming growth factor $\beta$ (TGF $\beta$ ) pathway, which plays key roles in cell proliferation, migration, extracellular matrix generation and epithelialmesenchymal transition [13]. The TGF $\beta$ pathway has been linked to several diseases related to altered fibroblastic function, most notably renal fibrosis and scleroderma, and the characteristic histological features, particularly the extracellular component of these tumours, have been attributed to the function of the EWSRI-SMAD3 chimeric protein [4].

In conclusion, we present the imaging and histopathological features of the first case of an EWSRI-SMAD3 fibroblastic tumour occurring in the bone. The lesion was treated with complete excision and has shown no signs of relapse over a prolonged (7 year) follow-up period. Chondroid differentiation, seen in this bone tumour, has not been described previously in soft tissue lesions. This tumour type is currently classified as benign in soft tissue, but the biological behaviour of the bone lesion cannot be inferred from a single case. We expand the spectrum of fibroblastic lesions of bone and add to the list of bone lesions showing EWSR1 gene rearrangement. The utility of powerful modern diagnostic techniques, such as whole-genome sequencing, is illustrated by this case report. Early recognition of this tumour type will facilitate appropriate management and increase the understanding of its biological behaviour.

Acknowledgements This research was made possible through access to the data and findings generated by the 100,000 Genomes Project; http:/ www.genomicsengland.co.uk.

Funding information Funding was provided by Sarcoma UK. The Tom Prince Cancer Trust and The Bone Cancer Research Trust.

\section{Compliance with ethical standards}

Conflict of interest The authors declare that they have no conflict of interest.

\section{References}

1. Baumhoer D, Amary F, Flanagan AM. An update of molecular pathology of bone tumors. Lessons learned from investigating samples by next generation sequencing. Genes Chromosomes Cancer. 2019:58(2):88-99.

2. Italiano A, Sung YS, Zhang L, et al. High prevalence of CIC fusion with double-homeobox (DUX4) transcription factors in EWSR1negative undifferentiated small blue round cell sarcomas. Genes Chromosomes Cancer. 2012;51(3):207-18.

3. Thway K, Fisher C. Mesenchymal tumors with EWSR1 gene rearrangements. Surg Pathol Clin. 2019;12(1):165-90. 
